\title{
Epidemiology of qnrVC alleles and emergence out of the Vibrionaceae family
}

The quinolones are antibiotics effective in the treatment of several current nosocomial infections. Bacteria carrying qnr genes present with decreased susceptibility to fluoroquinolones. In addition to this low-level resistance, qnr genes are associated with reduced bactericidal activity of ciprofloxacin in vitro and in vivo, which represents a therapeutic threat (Allou et al., 2009). Moreover, in association with mutations in gyrA/parC and with efflux pump regulatory systems, a full fluoroquinolone resistance phenotype can easily emerge, resulting in treatment failure (RodríguezMartínez et al., 2011). Recently, due to increasing reports of qnrVC alleles in different genetic contexts, these genes were classified within a new transferable $q n r$ family (Pons et al., 2013). Here, using in silico analysis, we show that qnrVC has already emerged worldwide out of the Vibrionaceae family in bacterial species of public health relevance, and in association with mobile genetic elements.

The qnrVC1 allele is a quinolone-resistance determinant that was first identified in a class 1 integron from a Brazilian Vibrio cholerae strain recovered in 1998, and was classified as a new and atypical member of the Qnr family (Fonseca et al., 2008). Different from the plasmid-mediated $q n r$ genes, which lack an $a t t C$ site (RodríguezMartínez et al., 2011), qnrVC1 was associated with a $V$. parahaemolyticus repeat (VPR), the recombination site responsible for the cassette mobilization characteristic of chromosomal integrons (Fonseca et al., 2008). Moreover, qnrVC1 carries its own functional promoter $\left(\mathrm{P}_{\mathrm{qnrVC}}\right)$ and its ability to confer decreased susceptibility to quinolones (MIC of ciprofloxacin, $0.25 \mu \mathrm{g} \mathrm{ml}^{-1}$ ) was determined (Fonseca et al., 2008; da Fonseca \& Vicente, 2012). Subsequently, this qnrVC1 cassette was identified in $V$. cholerae from Bangladesh and India in different genetic contexts, including SXT, which is a mobile element (Kim et al.,
2010; Kumar \& Thomas, 2011) (Table 1). Concurrently with qnrVC1 characterization the qnrVC2 allele was identified, by in silico analysis, in a plasmid from $V$. cholerae isolated in Vietnam (Fonseca et al., 2008). However, this gene is not functional because of the presence of several stop codons in its coding region and, consequently, does not contribute to the emergence of quinolone resistance.

Concomitantly to this qnrVC1 spread, new alleles were identified (Table 1). The qnrVC3 and qnrVC4 genes were also found in class 1 integrons from $V$. cholerae and Aeromonas punctata strains from India and China, respectively (Kumar \& Thomas, 2011; Xia et al., 2010), and their deduced proteins differed by four and 41 amino acids, respectively, compared with QnrVC1. The qnrVC4 gene was also found among environmental aquatic-borne species from other bacteria genera (Table 1). The $P_{q n r V C}$ promoter and the VPR recombination site from $q n r V C 1$ are conserved and identical in qnrVC3. However, despite the presence of the canonical $\mathrm{P}_{\mathrm{qnrVC}}$ in all qnrVC4 cassettes already described, their recombination sites correspond to different $V$. cholerae repeats (VCRs) (Table 1).

The qnrVC5 allele was recently identified in V. parahaemolyticus, V. cholerae and $V$. fluvialis from Haiti, China and India (Table 1). Some of these were erroneously annotated in GenBank as qnrB1 or qnrVClike, and here we have properly unified and named them as qnrVC5. The qnrVC5 gene differs by one non-synonymous mutation from qnrVC4 and by four indels from qnrVC2 (Fonseca et al., 2008). However, the qnrVC5 recombination site has the highest identity with the qnrVC2 VCR, and the plasmid from $V$. fluvialis, where qnrVC5 was found, showed $99 \%$ identity with pVN84 from $V$. cholerae $\mathrm{O} 1$ that harboured qnrVC2 (Fonseca et al., 2008; Rajpara et al., 2009). These data indicate that these two plasmids are the same
(Rajpara et al., 2009) and, considering the high similarity between $q n r V C 5$ and $q n r V C 2$ cassettes, we could hypothesize that $q n r V C 5$ is in fact the qnrVC2 functional form, and that qnrVC2, qnrVC4 and $q n r V C 5$ are closely related.

Altogether, these findings show that the qnrVC family has amazing mobility and dispersion through different hosts and environments, which suggests that the distinct recombination sites they are associated with (VPRs and VCRs; Table 1) have been effectively recognized and mobilized during site-specific recombination events.

To date, qnrVC alleles have been found in the Vibrionaceae family and among environmental aquatic-borne species. Moreover, qnrVC1 had until recently been identified only in $V$. cholerae. However, our GenBank search (March 2013) revealed the occurrence of the entire qnrVC1 gene cassette (including the 5 'UTR and the VPR site) in different class 1 integrons from carbapenem-resistant Pseudomonas aeruginosa strains found in Tunisia (Table 1) and a qnrVC-like allele in a class 1 integron array from Acinetobacter baumannii in China, two opportunistic pathogens responsible for nosocomial outbreaks worldwide. The QnrVC-like deduced protein had only one amino acid substitution (N71D) in comparison with QnrVC1. The putative $\mathrm{P}_{\mathrm{qnrVC}}$ presented one mismatch in the -35 hexamer compared with the canonical qnrVC1 promoter (da Fonseca \& Vicente, 2012), and the recombination site is also a VPR, although different from that of qnrVC1. Considering the polymorphism in the amino acid sequence and that a gene cassette is characterized by its attC site (Stokes et al., 1997), we can assume that this $q n r V C$-like is a new allele, named here qnrVC6 (Table 1).

Quinolones are clinically relevant antibiotics in the treatment of $P$. aeruginosa and other Gram-negative 
Table 1. Alleles of the qnrVC family

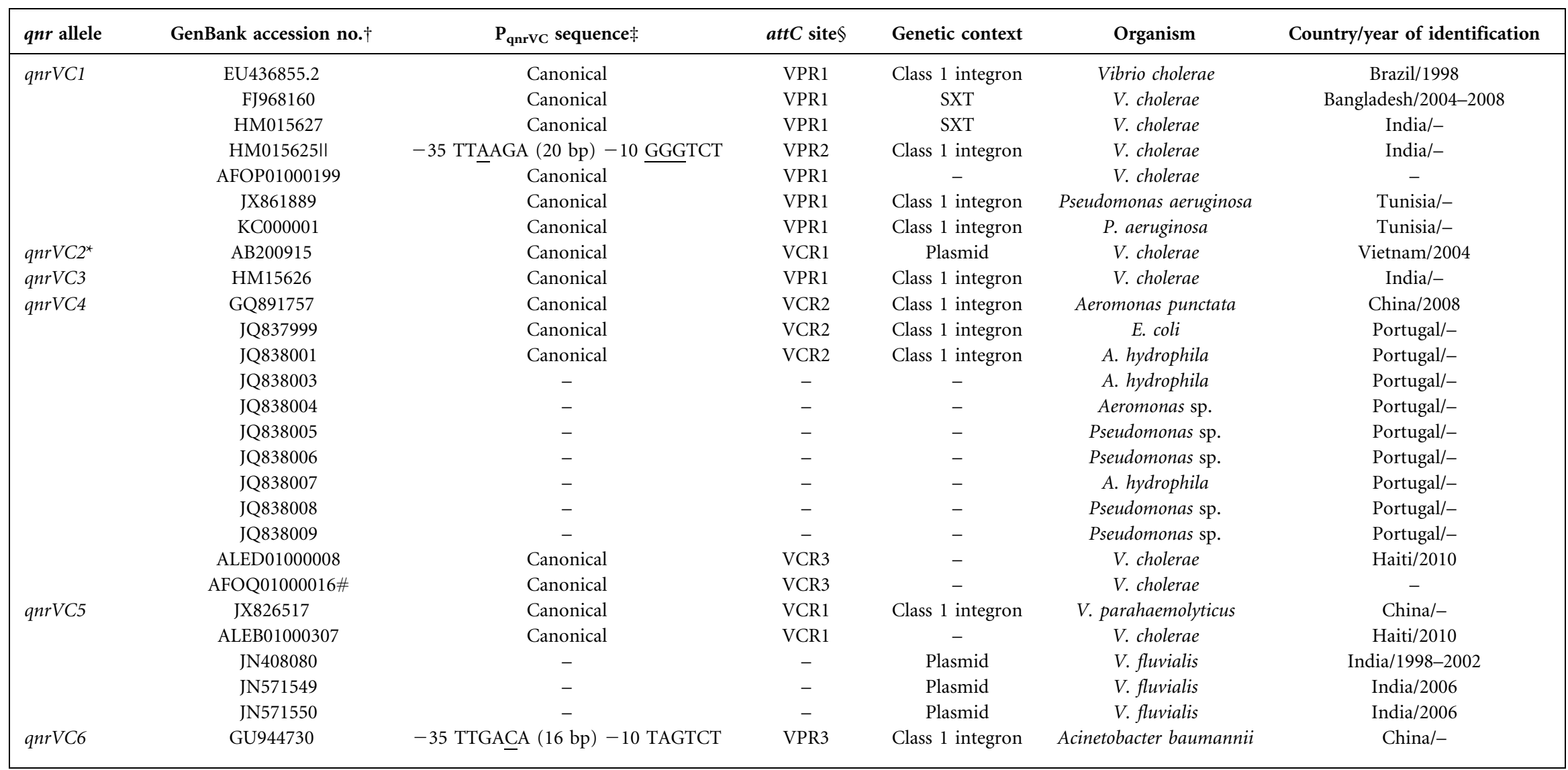

-, No sequence information provided; VCR, V. cholerae repeat; VPR, V. parahaemolyticus repeat.

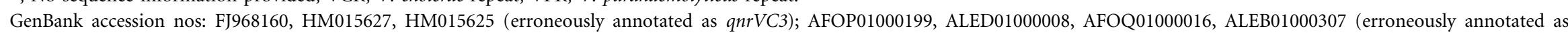
qnrB1); JN408080, JN571549, JN571550 (published as qnrVC-like without allelic definition; properly named here as qnrVC5).

${ }^{*} q n r V C 2$ is a non-functional allele because of the presence of premature stop codons.

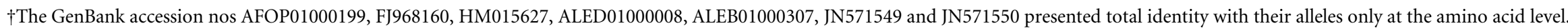
$\$$ Point mutations in $\mathrm{P}_{\mathrm{qnrVC}}$ hexamers are underlined.

$\S$ Numbers are included in order to distinguish different recombination sites.

IIGenBank accession no. HM015625 presents one silent mutation in the qnrVC1 coding region.

\#AFOQ01000016 presents one substitution relative to qnrVC4, but considering the conservation observed in the $5^{\prime}$ UTR and VCR, it remains assigned as a qnrVC4 allele. 
infections. Therefore, the presence of functional qnrVC genes in these species, contextualized in a clinic environment, is worrisome, since their expression and, consequently, the emergence of quinolone resistance in these strains becomes imminent.

\section{Acknowledgements}

This work was supported by a Fundação de Amparo à Pesquisa do Rio de Janeiro (FAPERJ) fellowship and Oswaldo Cruz Institute grant. The authors have no competing interests to declare.

\section{Erica L. Fonseca and Ana Carolina P. Vicente}

Laboratório de Genética Molecular de Microrganismos, Instituto Oswaldo Cruz, FIOCRUZ, Rio de Janeiro, Brazil

Correspondence: Erica L. Fonseca (ericafon@ioc.fiocruz.br)
Allou, N., Cambau, E., Massias, L., Chau, F. \& Fantin, B. (2009). Impact of low-level resistance to fluoroquinolones due to qnrA1 and qnrS1 genes or a gyrA mutation on ciprofloxacin bactericidal activity in a murine model of Escherichia coli urinary tract infection. Antimicrob Agents Chemother 53, 4292-4297.

da Fonseca, É. L. \& Vicente, A. C. (2012). Functional characterization of a cassette-specific promoter in the class 1 integron-associated qnrVC1 gene. Antimicrob Agents Chemother 56, 3392-3394.

Fonseca, E. L., Dos Santos Freitas, F., Vieira, V. V. \& Vicente, A. C. (2008). New qnr gene cassettes associated with superintegron repeats in Vibrio cholerae O1. Emerg Infect Dis 14, 11291131.

Kim, H. B., Wang, M., Ahmed, S., Park, C. H., LaRocque, R. C., Faruque, A. S., Salam, M. A., Khan, W. A., Qadri, F. \& other authors (2010). Transferable quinolone resistance in Vibrio cholerae. Antimicrob Agents Chemother 54, 799803.

Kumar, P. \& Thomas, S. (2011). Presence of qnrVC3 gene cassette in SXT and class 1 integrons of Vibrio cholerae. Int J Antimicrob Agents 37, 280-281.
Pons, M. J., Gomes, C. \& Ruiz, J. (2013). QnrVC, a new transferable Qnr-like family. Enferm Infecc Microbiol Clin 31, 191192.

Rajpara, N., Patel, A., Tiwari, N., Bahuguna, J., Antony, A., Choudhury, I., Ghosh, A., Jain, R., Ghosh, A. \& Bhardwaj, A. K. (2009). Mechanism of drug resistance in a clinical isolate of Vibrio fluvialis: involvement of multiple plasmids and integrons. Int J Antimicrob Agents 34, 220225.

Rodríguez-Martínez, J. M., Cano, M. E., Velasco, C., Martínez-Martínez, L. \& Pascual, A. (2011). Plasmid-mediated quinolone resistance: an update. J Infect Chemother 17, 149-182.

Stokes, H. W., O'Gorman, D. B., Recchia, G. D., Parsekhian, M. \& Hall, R. M. (1997). Structure and function of 59-base element recombination sites associated with mobile gene cassettes. $\mathrm{Mol}$ Microbiol 26, 731-745.

Xia, R., Guo, X., Zhang, Y. \& Xu, H. (2010). $q n r V C$-like gene located in a novel complex class 1 integron harboring the ISCR 1 element in an Aeromonas punctata strain from an aquatic environment in Shandong Province, China. Antimicrob Agents Chemother 54, 34713474. 